safely be left in the hands of a laboratory attendant, a procedure which I am convinced should never be adrocated even for the most simple manœuvres. In equally practised hands the method is, I believe, slower, more tedious, and more liable to accidental error than is the microscopic or other forms of the macroscopic methods.

The inaccuracies of the Dreyer method as at present employed are dealt with very exactly by Mr. R. Donald in THE LANCET of Sept. 2nd, 1916.1 These errors are essential errors of the technique, and $I$ will briefly recapitulate the more important of them. A quantitative agglutinin test depends for its accuracy upon the exactitude of the dilution of the serum to be tested. In the microscopic test these dilutions are made by means of a Wright's capillary tube and teat, an extremely accurate method which is quite unaffected by variations in size or diameter of the pipette. In the "standard" method the dilutions are made by drop-measuring, and the errors which Mr. Donald points out are actual errors proved by careful measurement. They are as follows. The "standard" pipettes issued are of different sizes. The only three pipettes examined by Mr. Donald differed seriously, and the difference in outer diameter of two pipettes issued specially for the performance of an extensive research differed in their drop count by as much as 20 per cent. The directions given for diluting the serum with saline make no allowance for the difference in drop count of serum and saline, and in making a 1 in 10 dilution of serum there is a further error of 10 per cent. A similar error, but this time of 20 per cent., occurs in the drop-measurement of the "standard" suspension. These errors may balance themselves or they may not; it is a matter of chance, and the same workers, however skilled, using the two standard pipettes with the directions given would at one time have an error of 40 per cent. and at another time none at all Now an error of 40 per cent. in measurement alone, when the measurements required can be made accurately by a simpler technique, is a very grave indictment of this "standard" method. It is true that the error is greatly reduced if one pipette is used throughout, but the facts are that the standard pipettes have been taken to be "standard," and have been used alternatively with such errors as are stated above, and if only one pipette is used the method becomes extraordinarily laborious.

While attacking the standard method in this manner, and in attempting to refute the attacks which have been made upon the microscopic method, I would like to confess that I have no doubts of the genuine idealistic views of the "standard" originators. I believe both methods have their minor faults and drawbacks, but I venture to support the view that we have not yet attained a method or a matériel, which warrants the general imposition of a fixed technique by a central authority.

The subsidiary question, none the less important, as to the behaviour of the agglutinin reaction in febrile inoculated patients, is one upon which I have little evidence to offer. I have looked up notes upon 100 consecutive cases from the Dardanelles. All of these patients had suffered from fever, the great majority being either paratyphoid or dysentery cases. All had been inoculated against typhoid at periods varying frorn one year to a few months previously. The typhoid agglutinin test was done in 72 of these cases. It was positive in 15 , or in 20.8 per cent. ; it was negative or partial - that is to say, gave a complete or partial agglutination in 1 in 20 dilutions and a partial or absent agglutination in 1 in 50 dilutions-in 16.7 per cent. It was completely negative - that is to say, it gave no reaction at all or only very slight clumping in 1 in 20 -in 62.4 per cent. $A$ $62 \cdot 4$ per cent. of completely negative tests in febrile cases who have been recently inoculated would seem to bear out the contention that the agglutinin content is diminished in such cases. I am unwilling, however, to include myself in the hymn of hate now being directed against Dr. H. L. Tidy on such evidence as I can offer. My cases are not sufficiently numerous, and they lack the evidence which 1 Since writing this paper I have read Dr. Ainley Walker's reply to
Mr. Donald in THE LANCET of Sept. $23 \mathrm{rd}$. The main inaccuracy is there eliminated on the supposition that only one pipette is used. As I point out, the use of one pipette renders the method extremely tedious, and certainly many users of the method have not tremely tedious, and certainly many users of the method have not confined as "the" pipette pipette. The directions, in alluding to the pipette as "the pipette, cannot seriously be considered to convey any appears to me advisable-namely, the proof of the amount of agglutinin set up by the inoculation and present before the fever.

- This paper would seem to be an attack upon the prominent supporters of the "standard "method; it is not so in reality. I have no doubt that the method as they use it gives reliable results, otherwise they would not advocate it. I merely wish to issue a plea that $I$ and many others may be permitted to use different methods which in our opinion and experience are equally reliable. The whole dispute has arisen out of the very laudable desire to correlate the research of a number of workers. If such correlation were possible by the adoption of a standard method, and could be done only in this way, it would be invidious to criticise it. Yet I believe a simpler and more harmonious plan would be to allow to the workers a choice of any of the recognised methods, in all of which the standard is known, and to leave to a central authority the conversion of those standards into equivalent results.

Queen Anne-street, W.

\section{A METHOD OF APPLYING THE WASSER- MANN REACTION IN LARGE NUMBERS.}

By P. FILDES, M.B., B.C. CantaB.,

ROYAL NAVAL HOSPITAL, HASLAR; ASSISTANT BACTERIOLOGIST TO THE LONDON HOSPITAL;

$$
\text { AND }
$$

JAMES MCINTOSH, M.D. ABERD., BACTERIOTOGICAL LABORATORY, LONDON HOSPITAL. (Both Working under the Medical Research Committee.)

ON several occasions recently we have been consulted upon the technique of the Wassermann reaction by those who propose to superintend or carry out these tests under the scheme promoted by the Local Government Board. Of recent years there have been few publications upon this subject, and therefore it may be opportune to give a detailed description of the technique without discussing the theory or rationale of the process in any way.

The details of the particular method which we describe are as introduced by one of us into the Royal Naval Hospital, Haslar. In this technique the reaction is reduced to the greatest possible simplicity. It is based upon that practised by us for several years, and which has been published in extenso. 1 The chief characteristic of our method is the use of a particular antigen composed of tissue extract mixed with cholesterin. Cholesterin was first used as an antigen by Fleischmann but apparently abandoned, and credit is due to Browning, Cruickshank, and Mackenzie for demonstrating first that cholesterin is capable of much increasing the specific action of another antigen. Browning and his co-workers amalgamated the cholesterin with "lecithin" extracted by themselves, but Sachs (1911) suggested the addition of small amounts of cholesterin to the organ extracts already in use.

In 1912 we published an extensive comparative test which we had carried out between various antigens, including Browning's and our own which we had founded upon the suggestions of Sachs. These tests showed that the formula worked out and suggested by us was more satisfactory than those hitherto in use and fulfilled the requirements of a " standard" antigen which would give comparable results in the hands of different workers. During the past four years this antigen has been extensively adopted, and is probably more widely used now than any other.

1. Collection of the serum to be tested. About $0.5 \mathrm{c} . \mathrm{c}$. of blood is required. This is very easily collected from the finger or thumb. The patient swings his right arm rapidly in order to drive the blood into* the finger tips; a piece of fairly thin drainage tubing is then twisted round the thumb and two or three punctures are made with a glass "pricker" or surgical needle just proximally to the nail and towards the ulnar side. The blood is collected in a Wright's capsule of suitable size. Fuse the straight end of the capsule. Attach a gummed label inscribed with the number of the case and enter up the particulars in the book under the 
same number. Put away the capsule into an efficient ice chest until required. ${ }^{2}$

At this point it may be strongly emphasised that it is of no value to spend time upon an elaborate Wassermann technique if the organisation permits of any possibility of mixing one serum with another. Grease pencil should never be used for writing numbers on glass. Every action involved in collecting the sample and "booking" it must be uninterrupted and rigidly according to routine.

2. Materials required for the test.

a. Copper racks to hold 24 test-tubes, as sold by Messrs. Baird and Tatlock, 14, Cross-street, Hatton-garden, E.C., for this purpose.

$b$. Test-tubes, $4 \times \frac{5}{8}$, washed and finally rinsed in distilled water and dried.

o. 1 c.c. and 10 c.c. graduated pipettes, specially long, graduated to tip (Baird and Tatlock).

d. Washed sheep's corpuscles. Collect the blood from a freshly killed animal into a stoppered bottle containing a few pieces of glass rod and iron wire. Fill only half full. Shake continuously for sufficiently long to defibrinate it. The blood will keep in the ice chest for three or four days. When required, fill the glass centrifuge buckets with the blood and drive down the corpuscles. Remove serum and add saline; shake and centrifuge. This constitutes the first washing. There must be three washings in all. The first may be carried out the day before the corpuscles are required. After the third washing remove the saline without shaking up the corpuscles.

$e$. Guinea-pig complement. On the day of the test kill a guinea-pig by culting its throat and collecting the blood in a saucer. The animal is first stunned stightly by knocking its head upon the edge of the table. Pour the blood from the saucer into a small conical glass. "Whip" to defibrinate with a piece of cotton-wool upon the end of an iron wire. Centrifuge and pipette off the serum.

$f$. Antigen. In two bottles A and B. Preparation of heart extract (A). Obtain a fresh human heart. With a pair of scissors cut off the muscular portions of the ventricles and elsewhere, but do not take fat. Mince these pieces and weigh them. Place in a mortar with a little sand and grind them up with absolute alcohol, using 9 c.c. of alcohol to $1 \mathrm{grm}$. of heart. Transfer the whole to a well-fitting glass-stoppered bottle, and shake orcasionally for one and a half hours. Filter into another well-fitting glass-stoppered bottle through paper and preserve in ice chest. A deposit will be found to form and this may be filtered off again. Cholesterin solution (B). Take 1 grm. of pure cholesterin, such as Kahlbaum's, and place in a well-fitting glass-stoppered bottle. Add 100 c.c. absolute alc nhol and put in the stopper tightly. Shake and heat in water bath until the cholesterin is dissolved.

g. Amboceptor. This will usually be purchased, although most sera upon the market are somewhat feeble in action.

3. Standardisation of the amboceptor.-To be performed with two different guinea-pigs to ensure maximum complement action. The standardisation should be repeated after several months.

a. Materials. 1. Saline solution. 2. Tube containing 0.5 c.c. guinea-pig's serum and 0.5 c.c. saline. 3 . Tube containing amboceptor diluted 1 in 1000 thus : Tube $a=$ ambo. $0.1 \mathrm{c.c}$. $(e x a c t)+$ saline 99 c.c. (shake); tube $b=1$ c.c. from tube $a+90$ c.c. saline (shake) - this is 1 in 1000 . 4. Tube containing 1 c.c. of deposited washed corpuscles +19 c.c. saline (shake). 5 Rack with 9 test-tubes $4 \times \frac{5}{8}$

b. Method. Fill reagents into the tubes as indicated.

\begin{tabular}{|c|c|c|c|c|c|c|c|c|c|c|}
\hline No. of test-tube ... & 1 & 2 & 3 & 4 & 5 & 6 & 7 & 8 & 9 & Pipette to \\
\hline 1. Saline $\ldots$... & 0.8 & 0.7 & 0.6 & 0.5 & 0.4 & $0 \cdot 3$ & $0 \cdot 2$ & $0 \cdot 1$ & 0 & 10 c.c. \\
\hline 2. $\underset{3 b)}{\operatorname{mb} b .} \quad$ (tube $\}$ & $0 \cdot 1$ & $0 \cdot 2$ & $0 \cdot 3$ & 0.4 & 0.5 & 06 & $0 \cdot 7$ & $0 \cdot 8$ & 0.9 & 1 c.c. \\
\hline $\left.\begin{array}{l}\text { 3. Complement } \\
\text { (tube } 2) \quad \ldots\end{array}\right\}$ & 0.1 & 0.1 & 01 & $0 \cdot 1$ & $0 \cdot 1$ & 0.1 & $0 \cdot 1$ & $0 \cdot 1$ & $0 \cdot 1$ & 1 c.c. \\
\hline 4. Blood (tube 4) & 0.5 & 0.5 & 0.5 & 05 & 0.5 & 0.5 & 05 & 0.5 & 0.5 & $10 \mathrm{c.c}$ \\
\hline
\end{tabular}

Shake. Incubate for one hour at $37^{\circ} \mathrm{O}$. and then read off the minimal hæmolytic dose-i.e., the first tube which is

2 If the ice chest is inefficient or if the sera have been sent by post or badly collected, it is better not to keep the capsules for several days but to centrifuge them at once and keep the serum separate from the corpuscles. absolutely clear. If the m.h.d. does not fall between 0.0001-0.0009 c.c. the test can be repeated, using the stronger dilution of amboceptor (tube $3 a$ ). It is better, however, to use an amboceptor which does fall between these limits.

4. Daily Routine.-(1) Wash or complete washing of the blood corpuscles.

(2) Kill the guinea-pig and prepare the serum.

(3) Make up fresh saline solution.

(4) Standardise the complement (A) and antigen control (B). (All subsequent measurements under 1 c.c. are made with the 1 c.c. pipette; up to 10 c.c. with the 10 c.c. pipette; above 10 c.c. with a 50 c.c. measure.) Materials: (a) Four tubes in the rack, front row, for $A$; four tubes in the rack, back row, for B. (b) Complement diluted 1 in 24 $(0 \cdot 1$ c.c. complement +2.3 c.c. saline). (c) Complement $2 \frac{1}{2}$ times stronger $(0.2$ c.c. complement +1.7 c.c. saline) for antigen control. (d) 5 per cent. corpuscle suspension con. taining amboceptor. This is made up as follows: The quantity required is 0.5 c.c. for every case to be tested +10 c.c. Thus if 60 bloods are to be tested, $30+10=40$ c.c. is required. The quantities will be as follows : (3) corpuscles (centrifuged deposit), 2.0 c.c. ; (2) amboceptor, 4 m.h.d. per tube-i.e., if m.h.d. $=0.0005,0.002$ per tube $\times 80=0.16$ c.c. ; (1) saline 37.84 c.c. Every half c.c. of this mixture will contain 0.002 of amboceptor, the correct quantity per tube. Make up in the order of the numbers and shake after each addition. (e) Diluted antigen, made up as follows:-Rinse out and clean a 1 c.c. pipette with spirit. Take 0.3 c.c. of heart extract and 0.2 c.c. of cholesterin and place in a dry test-tube. Run in 7 c.c. of saline and shake.

Method : fill in the 8 tubes in the rack as indicated.

Front Row (d) for Estimating m.h.d. of Complement.

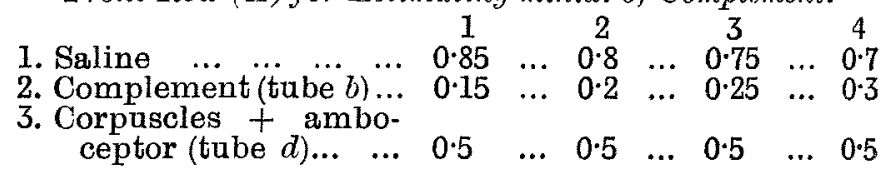

Back Row (B) for Antigen Control.

$\begin{array}{llllllllllll}\text { 1. Saline } & \ldots & \ldots & \ldots & \ldots & 0.35 & \ldots & 0.3 & \ldots & 0.25 & \ldots & 0.2\end{array}$

$\begin{array}{lllllllll}\text { 2. Complement }(\text { tube } c) \ldots & \ldots .15 & \ldots & 0.2 & \ldots & 0.25 & \ldots & 0.3\end{array}$

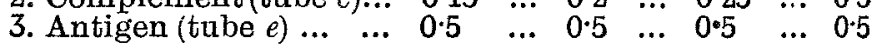

Shake. Put rack into a water bath at $37^{\circ} \mathrm{C}$. for 10 minutes to see whether the front-row tubes are laking properly, and then transfer to the incubator to complete 1 hour. Read the m.h.d. of complement in the front row and put 0.5 c.c. of corpuscles (tube $d$ ) into each of the back-row tubes. Put into the water bath and note whether the tube behind the m.h.d. tube is laked (about 15 minutes or less). If so, continue as in following table:-

If m.h.d. is tube 1 use 0.5 c.c. of a 1 in 33 dilution of complement for

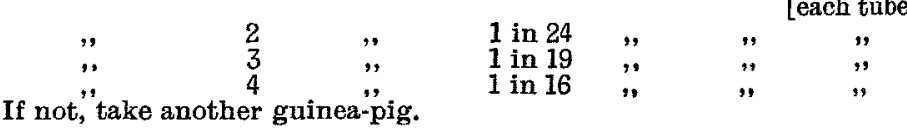

5. Preparation of serum for testing. - While the complement is undergoing standardisation, or at any other time on the day of the test, the sera should be distributed into the tubes. The numbered capsules are centrifuged and a series of test-tubes are set out in the racks, each numbered to correspond to the capsules. The numbering of the tubes must be indelible, and is best carried out by Donald's method. 1. Warm the tube in the flame. 2. Inscribe a bold figure on it with ordinary blue-black ink. 3. Burn in the ink by heating in the flame to a point short of the fusing temperature. From each capsule transfer 0.1 c.c. of clear serum to the corresponding tube. This is conveniently done with Donald's dropping pipettes, thus:-1. Pull out a piece of glass tubing into two pipettes. 2. Pass the pipette through a particular hole in a wire gauge plate, when it engages cut it off flush with the plate. The actual hole to be used is found once and for all by experiment. A pipette made in No. 53 Stubbs will deliver 0.1 c.c. of serum in 4 drops. Always hold the pipette vertically and wash with water between each serum. When the sera are all filled in make the tubes into a bundle in some safe and convenient manner and suspend them in a water-bath standing at $55^{\circ}-56^{\circ} \mathrm{C}$. for 30 minutes. Then replace them in the racks in sequence. When testing cerebro-spinal fluid use twice as much and do not heat it. 
6. Preparation of the reagents for use.-a. When the complement is standardised make up a sufficient quantity of the correct dilution to allow 0.5 c.c. for each tube and a little over. $b$. Also make up exactly the same quantity of diluted antigen as shown in the table. Mix in the manner already described for antigen.

\begin{tabular}{|c|c|c|c|c|c|c|c|c|}
\hline No. of tul & & C.c. & & Heart. & & oleste & & Saline \\
\hline 30 & $=$ & 15 & $=$ & 0.6 & + & 0.4 & + & $14 \cdot 0$ \\
\hline 40 & $=$ & 20 & $=$ & 0.8 & + & 0.53 & + & 186 \\
\hline 50 & $=$ & 25 & $=$ & $1 \cdot 0$ & + & 0.67 & + & $23 \cdot 3$ \\
\hline 60 & $=$ & 30 & $=$ & $1 \cdot 2$ & + & 0.8 & + & $28 \cdot 0$ \\
\hline 70 & $=$ & 35 & $=$ & $1 \cdot \overrightarrow{4}$ & + & 0.93 & + & $32 \cdot 6$ \\
\hline 80 & $=$ & 40 & $=$ & $1 \cdot 6$ & + & 1.06 & + & $37 \cdot 3$ \\
\hline 90 & $=$ & 45 & $=$ & $1 \cdot 8$ & + & $1 \cdot 2$ & + & $42 \cdot 0$ \\
\hline 100 & $=$ & 50 & $=$ & $2 \cdot 0$ & + & $1 \cdot 34$ & + & $46 \cdot 6$ \\
\hline 110 & $=$ & 55 & $=$ & $2 \cdot 2$ & $t$ & $1 \cdot 47$ & + & \\
\hline 120 & $=$ & 60 & $=$ & $2 \cdot \overline{4}$ & + & $1 \cdot 6$ & + & \\
\hline 130 & $=$ & 65 & $=$ & $2 \cdot 6$ & + & $1 \cdot 73$ & + & \\
\hline 140 & $=$ & 70 & $=$ & $2 \cdot 8$ & + & $1 \cdot 86$ & + & 65 \\
\hline 150 & $=$ & 75 & $=$ & $3 \cdot 0$ & + & $0 \cdot 0$ & + & \\
\hline
\end{tabular}

7. The test proper.-Mix the dilated complement and antigen together, and then, without delay, measure 1 c.c. of the mixture into every tube. Shake. Incubate for one hour in the air incubator. Add 0.5 c.c. of the corpuscle-amboceptor mixture to each tube. Place the racks in the waterbath and read results when some are quite laked and others quite opaque-i.e., in about 10 minutes.

8. Reading results. - When judging between complete inhibition and slight hæmolysis, depend more upon opacity than colour. Describe the inhibiting sera as ++++ (complete) $+++;++$ or.+++++ and +++ will be diagnostic of syphilis, ++ and + will be more or less doubtful when the tester has no personal knowledge of the case. If, however, the case is known to have had syphilis, ++ and + assume much more importance. The great majority of cases give ++++ or 0 .

\section{THE OCCASIONAL ABSENCE OF A RISE OF TEMPERATURE FOLLOWING THE ADMINISTRATION OF DIAGNOSTIC DOSES OF TUBERCULIN TO TUBERCULOUS PERSONS.}

By DUNCAN FORBES, M.D., B.Sc. EdIN., D.P.H , IEDICAL OTTICER OF HEALTH OF BRIGHTON; AND

C. W. HUTT, M.A., M.D. OANTAB., D.P.H, SENIOR SCHOOL DOCTOR AND ACTING TUBEROULOSIS OFFTCER, BRIGHTON

IT is well recognised that a local reaction and rise of temperature following the administration of tuberculin does not necessarily indicate the presence of an active lesion, signs of a focal reaction alone giving any evidence likely to be of assistance in the diagnosis. On the other hand, many believe that active disease can definitely be excluded if the usual diagnostic doses of tuberculin are not followed by any marked rise of temperature. Our reason for sending these few notes is to emphasise the fact that patients suffering from active tuberculosis and who have not been previously treated with tuberculin may have no rise of temperature of even $1^{\circ} \mathrm{F}$. above normal following the administration of diagnostic doses of tuberculin. In other words, the absence of a temperature reaction after the administration of maximum diagnostic doses of tuberculin does not prove the absence of active tuberculosis.

CASE 1.-The patient, a male aged 44, had a history of cough on and off for ten years, more marked since November, 1913. In July, 1914, he was admitted to the sanatorium for observation. He had received no tuberculin treatment prior to admission. There was impaired resonance at the right apex, but no sign of active disease. As some doubt existed as to the diagnosis, a short time after admission, at intervals of three and four days, 0.00002 P.T.O. and $\frac{1}{5}, \frac{1}{2}, 1,2$ 5 , and 10 milligrammes of tuberculin (T.) were given. The four-hourly chart showed no rise of temperature, except on the day following the $\frac{1}{2}$ and 1 milligramme doses, when the mouth temperature rose to $98.6^{\circ}$ and $99 \cdot 2^{\circ} \mathbf{F}$.; on two occasions prior to the administration of tuberculin a temperature of $99.2^{\circ}$ had been recorded. The local reaction was slight. The patient's weight steadily rose from $55^{\circ} 5$ kilos on July 17 th to 60 kilos on August 20 th, when he was discharged. A trace of albumin but no tuberole bacilli were found in his sputum at that time.

The patient's condition remained stationary until November, 1915, some 15 months later, when he was readmitted with definite crepitations at the right apex and tubercle bacilli in the sputum. On Dec. 20th, 28th and 31 st, and Jan. 6th $\frac{1}{5}, \frac{1}{2}, 1$, and 5 milligrammes of tuberculin (T.) were administered, followed by no increase of temperature over the normal, except on two occasions, $99^{\circ}$ being recorded on the third day after the $\frac{1}{2}$ and 5 milligramme doses; a temperature of $99^{\circ}$ had been recorded on Dec. 11th a short time prior to the injections. On Jan. 9th there was increase of cough and sputum but no rise in temperature. The sputum was then sent to the Lister Institute for examination in order to exclude the possibility that saprophytic acid-fast organisms had been mistaken for tubercle bacilli the guinea-pigs inoculated showed macroscopic signs of tuberculosis.

This case seems to prove that the routine diagnostic doses of tuberculin may not be followed by a temperature appreciably over the patient's normal temperature $(a)$ at an early stage of the disease when tubercle bacilli cannot be found in the sputum, and $(b)$ at later stages when these are present and even when a focal reaction indicated by increase of cough and sputum is caused.

CASE 2.-The patient, a male aged 2, was admitted suffering from spinal caries and healed tuberculous abscesses on the foot. He received $\frac{1}{2}, \frac{1}{2}$, and 1 milligramme of tuberculin (T.), the highest subsequent temperature being $98 \cdot 6^{\circ} \mathbf{F}$. Five months later an abscess formed on the dorsum of the foot, broke down, and remained open for some time. In this case the maximum dose administered was 1 milligramme, but the child was only 2 years of age.

In this connexion it is interesting to note that of the animals infected with human tubercle bacilli and tested with homologous tuberculin the following gave a temperature reaction $^{1}$ (rises of $0.90 \mathrm{O}$. over the normal): 107 of 124 calves, or 86.3 per cent. ; 6 of 6 goats, or 100 per cent. 10 of 11 pigs, or 90.9 per cent.; and 4 of 4 horses, or 100 per cent. Evidently neither in man nor in animals does the absence of a temperature reaction following tuberculin exclude active tuberculosis.

Brighton.

\section{Shedical Socreties.}

\section{ROYAL SOCIETY OF MEDICINE.}

\section{SECTION OF ELEOTRO-THERAPEUTICS.}

Exhibition of Case and of Skiagrams. - Gas Gangrene.

A MEETING of this section was held on Oct. 20th, Dr. G. Harrison Orton, the President, being in the chair.

Dr. E. P. Cumberbatch showed a female patient, about 60 years of age, for the purpose of illustrating what could be accomplished by the diathermic cautery in cases of malignant disease. In 1913 she came to him with a rodent ulcer on the scalp, measuring 1 by $\frac{3}{4}$ in. in diameter. Zinc ionisation, repeated six times, produced no benefit; neither did a course of $\mathrm{X}$ ray treatment. A combination of both those methods was equally barren of results, for at the end of 10 months the diameter of the ulcer had increased to $1 \frac{3}{4}$ by $1_{\frac{1}{3}}$ in. Accordingly, in March, 1914, he commenced the use of the diathermic cautery, cauterising with it the edges of the ulcer and the granulations at the base. There could now be seen a central area of parietal bone occupying the base of the ulcer, and around that the tissue was apparently healthy. That greatly improved condition had persisted for a year and seven months. In treating bone cases Dr. Cumberbatch insisted on great care being taken not to allow the diathermy to damage the periostenm. - Dr. W. J. TURRELL, in discussing the treatment, said that no attempt should be made to treat the whole growth at once, when the wound was a large one, but that treatment should be proceeded with in stages, so that the effect of the previous application could be assessed before entering upon another.

Dr. H. A. ECcles showed, by means of the epidiascope, a valuable series of skiagrams of Fractured Jaws, from a

1 Final Report of Royal Commission on Tuberculosis, Part II Appendix Report on Tuberculin Tests. 